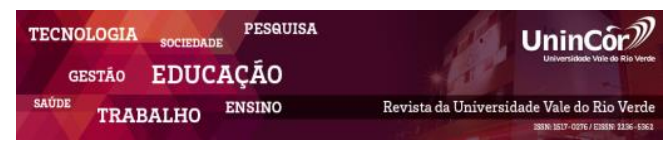

Revista da Universidade Vale do Rio Verde ISSN: 1517-0276 / EISSN: 2236-5362 v. $17 \mid$ n. 1 | Ano 2019

Lauana Aparecida Santos Doutoranda do Programa de Pós-graduação em Ciências Farmacêuticas da Universidade Federal de Alfenas - UNIFAL

lauanasantos20@gmail.com

Julianne Caravita Grisolia Doutoranda do Programa de Pós-graduação em Biociências Aplicadas à Saúde da Universidade Federal de Alfenas - Unifal juliecg@uol.com.br

Adriano Macedo de Oliveira Médico Patologista e Professor da Universidade José do Rosário Vellano - UNIFENAS adrianomacedo@unifenas.br

\section{PARACOCCIDIOIDOMICOSE: OS DESAFIOS DO DIAGNÓSTICO E TRATAMENTO}

\section{RESUMO}

Paracoccidioidomicose (PCM) é uma micose sistêmica causada pelo fungo termo-dimórfico Paracoccidioides brasiliensis. Este fungo apresenta-se na forma micelial na temperatura de $25^{\circ} \mathrm{C}$ sendo está produtora dos esporos ou conídios infectantes que, inalados pelos hospedeiros susceptíveis transformam-se em células leveduriformes nos tecidos esta forma pode ser observada na temperatura de $37^{\circ} \mathrm{C}$. Na observação da microscopia direta as células leveduriormes se apresentam em diferentes tamanhos. Está transição do processo morfogenético é reversível, e está relacionado a capacidade de adaptação às mudanças das condições ambientais. Esta micose apresenta maior número de casos em regiões com áreas tropicais e estando distribuída na América Central e do Sul. A paracoccidiodomicose possui diversas classificações de suas formas clínicas. Já o diagnóstico da PCM pode ser difícil de ser feito pois, na forma aguda e crônica da doença podem ser confundidos com outras doenças que apresentam os aspectos clínicos semelhantes. P. brasiliensis é susceptível à maioria das drogas antifúngicas, desta forma, vários antifúngicos podem ser utilizados no tratamento. No entanto, a grande problemática é o tratamento prolongado que muitas das vezes ocorrem o abandono. O objetivo deste trabalho é apresentar os métodos de diagnóstico e tratamento utilizados na clínica e os estudos direcionados no intuito de abreviar o tratamento desta doença. A partir dos dados da literatura é notório observar que o diagnóstico e tratamento são um desafio, uma vez que os sintomas podem ser confundidos com outras doenças e o tratamento prolongado dificulta um sucesso terapêutico.

Palavras-chave: Paracoccidioidomicose. Diagnóstico.

Tratamento.

\section{PARACOCCIDIOIDOMYCOSE: THE CHALLENGES OF DIAGNOSIS AND TREATMENT}

\begin{abstract}
Paracoccidioidomycosis (PCM) is a systemic mycosis caused by the thermo-dimorphic fungus Paracoccidioides brasiliensis. This fungus is present in the mycelial form at $25^{\circ} \mathrm{C}$ and is producing the infecting spores or conidia which, inhaled by the susceptible hosts, transform into yeast cells in the tissues. This form can be observed at $37^{\circ} \mathrm{C}$. In the direct microscopy observation the yeast cells present in different sizes. This transition from the morphogenetic process is reversible, and is related to the ability to adapt to changing environmental conditions. This mycosis presents the highest number of cases in regions with tropical areas and is distributed in Central and South America.
\end{abstract}


Paracoccidiodomycosis has several classifications of its clinical forms. The diagnosis of PCM can be difficult to do because in the acute and chronic form of the disease can be confused with other diseases that present similar clinical aspects. P. brasiliensis is susceptible to most antifungal drugs, in this way, various antifungals can be used in the treatment. However, the major problem is the prolonged treatment that often occurs the abandonment. The objective of this work is to present the methods of diagnosis and treatment used in the clinic and the studies aimed at abbreviating the treatment of this disease. From the data in the literature it is noteworthy to observe that diagnosis and treatment are challenging, since symptoms can be confused with other diseases and prolonged treatment makes therapeutic success difficult.

Keywords: Paracoccidioidomycosis. Diagnosis. Treatment.

Recebido em: 14/01/2019 - Aprovado em: 20/03/2019 - Disponibilizado em: 15/07/2019

\section{INTRODUÇÂO}

Paracoccidioides brasiliensis é um fungo termo-dimórfico agente etiológico da micose sistêmica paracoccidioidomicose (PCM). Este fungo foi descrito primeiramente por Adolfo Lutz como Coccidioides immitis que em 1930 após estudos de sua morfologia foi definido um novo gênero chamado Paracoccidioides. Durante 75 anos foi considerado apenas a espécie $P$. brasiliensis. Todavia, após estudos de distribuição geográfica e genéticos foram observados entre os isolados que o gênero é composto por um complexo de espécies.

Apesar de muitos estudos envolvidos nos aspectos biológicos do fungo e fisiopatologia da doença e tratamento, pouco se sabe sobre o habitat exato deste microrganismo. Este fungo já foi isolado do solo, de ração de cães e fezes de pinguim e morcegos. Estudos também descreveram que conseguiram isolar o fungo de órgãos de tatus (Dasypusn ovemcinctus e Cabassous centralis) e recentemente em animais silvestres e domésticos (CANTEROS, 2018). As características do habitat deste fungo estão associadas e reconhecidas devido aos casos clínicos ocorrerem com pessoas que habitam ou viveram em algum momento de sua vida em locais com florestas úmidas e que exercem atividades agrícolas.

A infecção pode ocorrer em seres humanos e animais a partir da inalação de conídios do fungo. Não há relatos da transmissão da PCM ocorrer de animais para as pessoas e de entre as pessoas em si. A princípio frequentemente esta infecção pode ser assintomática. A paracoccidioidomicose tem como característica a presença de granulomas nos tecidos dos indivíduos acometidos. Esta doença tem como a principal via de infecção o trato respiratório que inicialmente este microrganismo atinge os pulmões e podendo disseminar-se para vários órgãos. Os indivíduos que apresentam a forma aguda desta micose é a mais grave e com um prior prognóstico. 
O diagnóstico desta micose consiste na visualização de células fúngicas isolados de fluídos biológicos. Para avaliação da gravidade e seguimento do tratamento os testes sorológicos são mais utilizados em destaque o ensaio de imunodifusão radial dupla. A utilização do diagnóstico por biologia molecular tem sido descrita em estudos, no entanto ainda não é aplicado na rotina laboratorial clínica (MENDES et al., 2017)

P. brasiliensis é susceptível à maioria das drogas antifúngicas, desta forma, vários antifúngicos podem ser utilizados para o tratamento de pacientes com PCM, tais como anfotericina B, sulfamídicos (sulfadiazina, associação sulfametoxazol/trimetoprim), azólicos (cetoconazol, fluconazol, itraconazol) (SHIKANAI-YASUDA, 2015). Desta forma, é de extrema importância conhecer a fisiopatologia, pois um de seus desafios é o diagnóstico e tratamento correto. O objetivo deste é artigo é apresentar os métodos de diagnósticos e fármacos utilizados em pacientes com PCM.

\section{MATERIAL E MÉTODOS}

A revisão de literatura consistiu da consulta, busca e leitura de banco de dados (Scielo, PubMed, Cochrane e outros) de artigos científicos. Os critérios de inclusão dos artigos selecionados foram trabalhos em diferentes idiomas que abordassem o conteúdo proposto e que tivessem sido publicados nos últimos oito anos.

\section{REVISÃO DE LITERATURA}

\subsection{Paracoccidioides brasiliensis e} Paracoccidioidomicose (PCM)

Paracoccidioides brasiliensis é um fungo termo-dimórfico agente etiológico da micose sistêmica paracoccidioidomicose (PCM) que pode apresentar-se na forma micelial na temperatura de $25^{\circ} \mathrm{C}$ sendo esta produtora dos esporos ou conídios infectantes que quando inalados pelos hospedeiros susceptíveis transformam-se em células leveduriformes nos tecidos esta forma pode ser observada na temperatura de $37^{\circ} \mathrm{C}$. Ao realizar o cultivo deste fungo na temperatura de $25^{\circ} \mathrm{C}$ observa-se que as colônias se apresentam após 15 a 30 dias de crescimento uma coloração esbranquiçada que vai se tornando acastanhada e aveludada. Na temperatura de $37^{\circ} \mathrm{C}$ e em tecidos de humanos e animais o crescimento deste fungo é lento. Ao realizar microscopia direta as células leveduriormes deste fungo se apresentam em diferentes tamanhos, sendo ovais, esféricas ou elípticas. Está transição do processo morfogenético é reversível, e está relacionado a capacidade de adaptação às mudanças das condições ambientais onde a conversão da forma micelial para levedura segue a cronologia da infecção, permitindo a sobrevivência do fungo e sua invasão nos tecidos do hospedeiro (SHIKANAI-YASUDA et al., 2017; MENDES et al., 2017).

Este fungo foi descrito primeiramente por Adolfo Lutz como Coccidioides immitis que em 1930 após estudos de sua morfologia foi definido um novo gênero chamado Paracoccidioides. Durante 75 anos foi considerado apenas a espécie 
P. brasiliensis. Todavia, após estudos de distribuição geográfica e genéticos foram observados entre os isolados que o gênero é composto por um complexo de espécies. No ano de 2009 identificaram duas espécies taxonômicas: P. brasiliensis sendo composto por quatro espécies denominadas de S1, PS2, PS3 e PS4 e o P. lutzii. Portanto, os agentes causadores da PCM são; $P$. brasiliensis (S1), $P$. americana (PS2), $P$. restrepiensis (PS3), P. venezuelensis (PS4) e $P$. lutzii (MUÑOZ et al., 2016; SHIKANAIYASUDA et al., 2017).

Apesar de muitos estudos envolvidos nos aspectos biológicos do fungo e fisiopatologia da doença e tratamento, pouco se sabe sobre o habitat exato deste microrganismo. Este fungo já foi isolado do solo, de ração de cães e fezes de pinguim e morcegos. Estudos também descreveram que conseguiram isolar o fungo de órgãos de tatus (Dasypusn ovemcinctus e Cabassous centralis) e recentemente em animais silvestres e domésticos (CANTEROS, 2018). As características do habitat deste fungo estão associadas e reconhecidas devido aos casos clínicos ocorrerem com pessoas que habitam ou viveram em algum momento de sua vida em locais com florestas úmidas, altas taxas de precipitação, exercício de atividades agrícolas, cursos de água e temperatura em torno de 17 e $24^{\circ} \mathrm{C}$. É de extrema importância conhecer o nicho ecológico deste fungo uma vez que pode implementar medidas de saúde pública no intuito de evitar a infecção primária (RESTREPO et al., 2012).

A infecção pode ocorrer em seres humanos e animais a partir da inalação de conídios do fungo. Não há relatos da transmissão da PCM ocorrer de animais para as pessoas e de entre as pessoas em si. A princípio frequentemente esta infecção pode ser assintomática e de acordo com levantamentos epidemiológicos $25 \%$ dos indivíduos que residem em áreas endémicas tem inoculação intradérmica positiva a reação a paracoccidioidina. A infecção a este fungo pode permanecer assintomática por anos e produzir uma doença ativa após este longo período de infecção primária. $\mathrm{O}$ início dos sintomas pode ocorrer por estilo de vida como alcoolismo, fumo má nutrição ou até mesmo imunossupressão. A princípio os indivíduos acometidos por esta micose apresentam tosse, dor no peito, febre, mal-estar e, em casos graves hemoptise, falta de ar e febre alta. A PCM pode apresentar como uma doença progressiva aguda ou sub-aguda também denominada de forma juvenil que acomete crianças e adolescentes ou como uma doença crônica que geralmente acomete adultos (RESTREPO et al., 2012).

A paracoccidioidomicose tem como característica a presença de granulomas nos tecidos dos indivíduos acometidos. Esta doença tem como a principal via de infecção o trato respiratório que inicialmente este microrganismo atinge os pulmões e podendo disseminar-se para vários órgãos. Ao fungo atingir outros órgãos acaba originando lesões secundárias nas mucosas, linfonodos, pele e glândulas adrenais (FERNANDES et al., 2017). Nos indivíduos acometidos pode-se observar que são produzidos granulomas ulcerosos nas superfícies das mucosas oral, nasal, trato-gastrointestinal pele e sistema reticuloendotelial. Como em outras micoses a PCM depende da interação do fungo 
(neste caso deve-se levar em consideração a virulência da cepa) e a resposta imunológica do hospedeiro. A partir desta interação a doença pode apresentar-se como assintomática ou disseminar para o organismo (BORGES et al., 2002).

Os indivíduos que apresentam a forma aguda desta micose é a mais grave e com um prior prognóstico. Esta forma tem como característica um curso rápido. Ao realizar a biópsia pode observar um grande número de células fúngicas multiplicando e sem a formação de granulomas. Ao realizar também exame clínico-radiográfico o pulmão apresenta-se incialmente sem lesões especiais (SHIKANAIYASUDA et al., 2017). No entanto, na forma grave esta progride lentamente e são observados sintomas pulmonares evidentes, mas pode-se observar que as manifestações pulmonares podem ser silenciosas. Desta forma, o indivíduo somente procura atendimento médico quando há o aparecimento de lesões extrapulmonares e que neste caso o quadro já está instalado e é multifocal (PALMIEIRO et al., 2005).

\subsection{Epidemiologia da doença}

Micoses sistêmicas como a paracoccidioidomicose podem ocorrer predominantemente em regiões com climas específicos. Esta micose apresenta maior número de casos em regiões com áreas tropicais e estando distribuída na América Central e do Sul (LEE; LAU, 2017). O fungo $P$. brasiliensis pode ser encontrado em regiões muito úmidas no qual há a prevalência do cultivo da cana de açúcar e café e onde o solo apresenta-se com características ácidas. A PCM afeta trabalhadores rurais que estão expostos a contato intenso e contínuo com o solo. A doença tem predomínio nos homens e com menor incidência em mulheres devido à proteção conferida pelo hormônio estrogênio. Este hormônio inibe ou dificulta a transformação de conídios e fragmentos de micélio para a forma leveduriforme, que é patogênica. Esta micose apresenta maior número de casos entre indivíduos de 30 a 59 anos de idade e também entre os de raça mista (MENDES et al., 2017).

A PCM é prevalente nos países como Brasil, Colômbia, Venezuela, Paraguai e México. No Chile devido as condições climáticas desfavoráveis ao crescimento do fungo no solo é o único país Sul-Americano sem qualquer notificação autóctone. A problemática desta doença é em conta a falta de registros contínuos dos casos de PCM (BELLÍSSIMO et al., 2013; MARTINEZ, 2017). Desta maneira o número de indivíduos acometidos tem sido estimado tendo como base casos relatados. No Brasil esta estimativa é baseada além dos casos que são relatados também dados de hospitalização e mortalidade. Há relatos na literatura que $80 \%$ dos pacientes adquirem a doença no Brasil e o restante adquirem em outros países da América do Sul em destaque Colômbia, Venezuela, Argentina e Equador. No período de 1996 a 2006 tem-se um total de 168 mortes registrados por ano no Brasil de indivíduos com paracoccidioidomicose (BELLÍSSIMO et al., 2013).

Estudos epidemiológicos permitiram estimar os estados com maior incidência da PCM no Brasil. Os estados de Rio Grande do Sul, São 
Paulo e Rio de Janeiro apresentaram maior número de casos a cada 100.000 habitantes (MARTINEZ, 2017). Estudo sobre prevalência de tipos sanguíneos entre pacientes com PCM em função da gravidade da doença e comparando com indivíduos saudáveis, sugeriu que os antígenos dos glóbulos vermelhos Jka, Jkb, Fyb e Leb podem desempenhar um papel na imunopatologia da doença, possivelmente como fatores de resistência (MENDES et al., 2017).

\subsection{Diagnóstico da paracoccidioidomicose}

A paracoccidiodomicose possui diversas classificações de suas formas clínicas que são baseadas em diferentes critérios, como a topografia das lesões, história da doença, gravidade do quadro clínico, resultados de reações sorológicas, entre outros (SHIKANAI-YASUDA et. al., 2017). A classificação das formas clínicas e gravidade da doença utilizadas hoje em dia, foi apresentada no International Colloquium on Paracoccidioidomycosis realizado em fevereiro de 1986 em Medellín na Colômbia, onde a gravidade da PCM pode ser classificada como: Infecção paracoccidioidica, paracoccidioidomicose (doença), forma aguda/subaguda (juvenil), moderada, grave, forma crônica (do adulto), leve, moderada, grave e como forma residual ou sequelas (SHIKANAIYASUDA et al., 2006).

Normalmente o diagnóstico se dá pela identificação de presença de reação intradérmica positiva ao antígeno específico e achados de fungos latentes em necropsia (SHIKANAIYASUDA et al., 2006).
O diagnóstico da PCM pode ser difícil de ser feito pois, na forma aguda da doença ele pode ser confundido com linfoma, leucemia, histoplasmose, tuberculose, toxoplasmose, leishmaniose visceral e mononucleose infecciosa. Já na forma crônica da doença pode levar a diagnósticos conflitosos com doenças como leishmaniose cutânea ou mucosa, tuberculose, cromoblastomicose, hanseníase, sarcoidose e neoplasias. Quando ocorre um comprometimento pulmonar da doença ainda pode ser confundida com tuberculose, coccidioidomicose, histoplasmose, sarcoidose, pneumoconiose e pneumonite intersticial (SHIKANAI-YASUDA et. al., 2017).

O “padrão ouro" para o diagnóstico da PCM é a identificação de elementos fúngicos sugestivos de Paracoccidioides spp em exame a fresco de escarro ou outro espécime clínico (raspado de lesão, aspirado de linfonodos) ou fragmento de biópsia de órgãos acometidos (SHIKANAI-YASUDA et. al., 2017). As formas fúngicas encontradas podem ser células vazias ou colapsadas sugerindo que podem ser fungos que já se reproduziram. Quando os fungos estão na forma jovem e viáveis apresentam conteúdo denso, no entanto quando estes microrganismos estão mortos a coloração empregada apresentam conteúdo de tonalidade clara ou aparecem vazios, pois ocorreu perda de conteúdo citoplasmático.

Outras provas laboratoriais especificas como sorologia para PCM podem ser importantes para um diagnóstico mais preciso da doença, atualmente estão disponíveis em serviços de referência testes como de imunodifusão dupla (IDD), contraimunoeletroforese (CIE), ensaio 
imunoenzimático (ELISA) e imunoblot (IB) (DEL NEGRO et al., 2000; DO VALLE et al, 2001). Normalmente esses testes possuem uma sensibilidade e especifidade que pode variar de $80 \%$ a 95\%, onde os títulos de anticorpos encontrados podem indicar a gravidade da doença, dentre os testes sorológicos a reação de imunodifusão em gel é a mais específica, e de custo mais acessível, sendo mais viável para o uso de diagnóstico de rotina para PCM.+

Embora apresentem uma boa sensibilidade e especifidade para o diagnóstico da PCM, é importante ressaltar que alguns estudos demostram que os testes possuem uma baixa sensibilidade na investigação de infecções por $P$. lutzii (TEIXEIRA et al., 2014). Ainda podem produzir reações falso-positivas, onde podem reações cruzadas com histoplasmose, aspergilose e leishmaniose.

Recursos adicionais mais avançados para o diagnóstico da PCM foram desenvolvidos, como a técnicas de imunoblot, o teste de ELISA para triagem, a pesquisa de antígeno específico e técnicas de PCR, estas apresentam maior exatidão no diagnóstico, mas não estão disponíveis para a rotina de atendimento de PCM (SHIKANAIYASUDA et. al., 2017).

\subsection{Tratamento da paracoccidioidomicose}

P. brasiliensis é susceptível à maioria das drogas antifúngicas, desta forma, vários antifúngicos podem ser utilizados para o tratamento de pacientes com PCM, tais como anfotericina B, sulfamídicos (sulfadiazina, associação sulfametoxazol/trimetoprim), azólicos (cetoconazol, fluconazol, itraconazol) (SHIKANAI-YASUDA, 2015). Medicamentos como sulfamídicos e derivados tem sido usado desde 1940 e consiste em um tratamento longo, onde sua posologia é uma das desvantagens, pois pode causar efeitos adversos, como reações de hipersensibilidade, sintomas gastrintestinais, anemia hemolítica, além de ser altamente distribuído por todo o organismo, mesmo no sistema nervoso central (SNC), e 10-30\% dos pacientes com doença ativa pode ter lesões do SNC. Também são usados medicamentos azólicos (cetoconazol, fluconazol e itraconazol), o cetoconazol foi usado com sucesso no tratamento PCM, mas por possuir má absorção e possuir efeitos adversos como o aumento dos níveis de transaminases, reação de hipersensibilidade da pele, vômitos, náuseas e anorexia, foi substituído pelo itraconazol (GOLDANI; WIRTH, 2017).

O fluconazol pode ser utilizado de forma oral e intravenosa sua principal desvantagem é baixa eficácia em casos disseminados. Os efeitos adversos incluem náuseas, erupções cutâneas, vômitos, dor abdominal e diarreia. Já o itraconazol é considerado de 10 a 100 vezes mais ativo do que o cetoconazol em células fúngicas, possui uma meia vida de 17 a 21 dias permitindo a sua prescrição, uma vez por dia 99,9\% da droga se liga às proteínas do plasma, e não atravessa a barreira hemato-encefálica, desta forma são encontradas em baixos níveis no fluído cerebral. A anfotericina B tem uma meia-vida de alguns dias, podendo ser administrado em dias alternados, considerado um composto fungistático e fungicida altamente ativo, tem sido usada desde 1958 para o tratamento de casos mais graves de 
PCM. Anfotericina B é excretada na bile após metabolização hepática e pode causar efeitos adversos como, hipocalemia, acidose tubular renal, diminuição da filtração glomerular e anemia hipocrômica normocítica (SHIKANAIYASUDA, 2015).

O itraconazol é um antifúngico que possui um largo espectro, clinicamente utilizado para tratamento de uma grande de variedade de infecções fúngicas como aspergilose, blastomicose, coccidioidomicose, criptocose, histoplasmose, esporotricose, paracoccidioidomicose, entre outras. A utilização do itraconazol para a PCM foi demostrada tanto “'in vivo" como "in vitro'. No Brasil é encontrado somente na forma de uso oral em cápsulas, sendo que o itraconazol pode ser administrado em doses elevadas (400 mg por dia ou duas vezes por dia), pois os efeitos adversos são pouco frequentes nas doses recomendadas, ocorrendo, tais efeitos são facilmente controlados, incluem náuseas, vômitos, aumento das transaminases séricas, erupção cutânea, hipocalemia, hipotrigliceridemia e hiperuricemia (SHIKANAI-YASUDA, 2015).

De acordo com dados descritos em Shikanai-Yasuda (2002), o tratamento com itraconazol se mostra eficaz com menos tempo de duração do tratamento, outros estudos comparativos demonstram que o uso do itraconazol para o tratamento da PCM tem sido a droga de escolha na maioria dos casos (MENEZES; SOARES; FONTES, 2006). Estudos mostram que na maioria dos casos os azóis de ação rápida são alternativas terapêuticas boas nos casos das formas mais leves da doença.
Os casos moderados a graves necessitam de tratamentos mais prolongados. Estudos comparativos mostram que o uso do itraconazol é capaz de induzir cura em pacientes cronicamente infectados. Ainda existe a necessidade de busca por combinaçãoes e novas drogas capazes de modular uma resposta imune mais eficaz nos casos graves de PCM (GOLDANI; WIRTH, 2017).

Alguns relatos demostram que os antifúngicos podem interagir de maneira sinérgicas com os mecanismos microbicidas de polimorfonuclares (PMN), através de mecanismos diretos e regulados por citocinas inflamatórias, como no caso da anfoterina B. Estudos demostraram que em camundongos tratados com anfotericina B possuem um aumento na atividade fungicida de macrófagos, onde foi descrito como sendo um fator ativador de macrófagos, além de aumentar a produção de TNF- $\alpha$. Também foi demonstrado que a anfotericina $\mathrm{B}$ exerce efeitos moduladores sobre PMN, estimulando a produção de citocinas inflamatórias, aumentando a atividade fagocitária. Dados com relação a capacidade do itraconazol ser capaz de aumentar a eficiência da resposta de PMN, ou influenciar a resposta imune inata e adquirida ainda não são concretos, apesar de ser a droga de escolha no tratamento da PCM. Assim, considerando que o tratamento da paracoccidioidomicose é prolongado, e que o uso de medicamentos pode levar a efeitos colaterais e até mesmo o desenvolvimento de resistência a fungos, os estudos recentes utilizando a terapia a laser de baixa intensidade (LLLT) tem mostrado uma 
alternativa no tratamento da doença (GOLDANI; WIRTH, 2017).

A radiação LASER de baixa intensidade (LLLT) possui propriedades anti-inflamatórias. Possuem comprimentos específicos de onda, tendo a capacidade de alterar o comportamento celular. Os efeitos do laser de baixa intensidade na aceleração da cicatrização de feridas têm sido atribuídos a vários fatores, entre eles a modulação da produção dos fatores de crescimento e redução na produção de prostaglandinas. Alguns estudos relatam que o uso do LLLT pode atuar em processos intracelulares, onde a luz pode ser absorvida por componentes da cadeia respiratória mitocondrial, podendo resultar em aumento da produção de espécies reativas de oxigênio. Pode aumentar a produção de prostaglandina. Assim o LLLT pode ter um papel na terapia imunobiológica para as doenças do sistema imune podendo ativar e aumentar a reação normal dos componentes do sistema imunológico (MENDES et al., 2017).

Estudos demostraram que a irradiação com LLLT mostrou ter um efeito antiinflamatório e pró inflamatório de acordo com o local e o momento da irradiação. Desta forma dados na literatura mostram que camundongos infectados com $P$. brasiliensis nas patas e tratados com irradiação a laser baixa potência neste local, tiveram uma redução do edema de pata, apresentaram também uma cicatrização mais rápida das feridas e nenhuma forma de $P$. brasiliensis detectada no local, enquanto o grupo controle apresentou múltiplos fungos viáveis no local da infecção. Dados apresentados por Burger et al (2015) demostraram que o uso do LLLT em camundongos infectados por $P$. brasiliensis foi capaz de causar um aumento da capacidade fungicida e também permitiu recrutar PMN mais ativos, capazes de combater a cepa altamente virulenta $P$. brasiliensis (Pb18).

\section{CONSIDERAÇÕES FINAIS}

A partir dos dados encontrados na literatura é possível perceber os desafios do diagnóstico desta micose. Desta forma, pode-se considerar um dos fatores primordiais na dificuldade do tratamento desta doença é o diagnóstico definitivo. $\mathrm{O}$ diagnóstico muito das vezes pode partir de a dificuldade do médico não solicitar o exame correto e não compreendendo os aspectos clínicos apresentados por esta doença. Além disso, a grande dificuldade é em profissionais que saibam fazer a correta identificação das formas fúngicas nos materiais a fresco recebidos e em biópsias. Também é válido mencionar que um dos grandes desafios é que o tratamento é prologado e desta forma muita das vezes o paciente pode abandona-lo e dificultando ainda mais o sucesso terapêutico. Estudos tem sido realizado com o intuito de abreviar o tempo de tratamento e assim evitar que o paciente abandone o uso dos medicamentos. Programas de disseminação descrevendo o que é a doença e formar de identificar e tratar podem reduzir estes desafios e também programas de prevenção com intuito de informar a população sobre a possível ocorrência desta micose e a importância de procurar assistência médica. 


\section{REFERÊNCIAS}

BELLÍSSIMO R. et al. Martinez, R. Endemic paracoccidioidomycosis: Relationship between clinical presentation and patients' demographic features. Med. Mycol. v.51, p.313-318, 2013.

BORGES W.M.I., CHEN D., SHU X., WALMSLEY A.R. The pathobiology of Paracoccidioides brasiliensis. Trends Microbiol. v.10, p.8087,2002 .

BURGER, E. et al. Low-level Laser Therapy to the Mouse Femur Enhances the Fungicidal Response of Neutrophils against Paracoccidioides brasiliensis. PLoS Neglected Tropical Diseases. v. 9, p. e0003541-1-19, 2015.

CAMARGO Z.P., BERZAGHI R., AMARAL C.C., SILVA S.H. Simplified method for producing Paracoccidioides brasiliensis exoantigens for use in immunodiffusion tests. Med Mycol. v.41, n.6, p.539-42, 2003.

CANTEROS C.E. PARACOCCIDIOIDOMICOSIS: CRÓNICA DE UNA ENFERMEDAD OLVIDADA. MEDICINA (Buenos Aires). v.78, p. 180-184, 2018.

DEL NEGRO G.M., et al. Evaluation of tests for antibody response in the follow-up of patients with acute and chronic forms of paracoccidioidomycosis. J Med Microbiol. v.49,n.1, p.37-46,2000.

DO VALLE A.C., et al. Interpretation and clinical correlation of serological tests in paracoccidioidomycosis. Med Mycol. v.39, n.4,p.373-7, 2001.

FERNANDES F.F., et al. The impact of paracoccin gene silencing on Paracoccidioides brasiliensis virulence. mBio 8:e00537-17. https://doi.org/10.1128/mBio.00537-17. Invited Editor Christina A. Cuomo, 2017.

GOLDANI LZ, WIRTH F, Animal Models and Antifungal Agents in Paracoccidioidomycosis: An Overview. Mycopathologia 2017, Aug, 182 (78):633-64.

LEE P.P., LAU Y.L. Cellular and Molecular Defects Underlying Invasive Fungal Infections-
Revelations from Endemic Mycoses. Front.

Immunol. v.8, p.735, 2017.

MARTINEZ R. New Trends in

Paracoccidioidomycosis Epidemiology. J. Fungi. v.3, n.1; p.2-13, 2017.

MENDES, R.P. et al. Paracoccidioidomycosis: Current Perspectives from Brazil. The Open Microbiology Journal, v.11, p.224-282, 2017.

MENEZES, V.M.; SOARES, B.G.; FONTES, C.J; Drugs for treating paracoccidioidomycosis. Cochrane Database Syst. Rev., 2006.

MORETO T.C., et al. Accuracy of routine diagnostic tests used in paracoccidioidomycosis patients at a university hospital. Trans R Soc Trop Med Hyg.v.105, n.8, p.473-8, 2011.

MUÑOZ J.F. et al. Genome diversity, recombination, and virulence across the major lineages of Paracoccidioides. mSphere. v.1n.5, p.00213-16, 2016.

PALMIEIRO M.; CHERUBINI K.; YURGEL L.S.; Paracoccidioidomicose - Revisão da Literatura; Scientia Medica, v.15, n. 4, p. 274278, 2005.

RESTREPO A., GÓMEZ B.L., TOBÓN A. Paracoccidioidomycosis: Latin America's own fungal disorder. Curr Fungal Infect Rep. v.6, p. 303-11, 2012.

SHIKANAI-YASUDA M.A., et al. Brazilian guidelines for the clinical management of paracoccidioidomycosis. Rev Soc Bras Med Trop. v.50, n.5, p.715-740, 2017.

SHIKANAI-YASUDA M. A; Paracoccidioidomycosis Treatment. Rev. Inst. Med. Trop. v. 19, n.57, p. 31-37, 2015.

SHIKANAI-YASUDA M.A., et al; Guideliness in paracoccidioidomycosis; Revista da Sociedade Brasileira de Medicina Tropical. v.39, n.3, p.297-310, 2006.

TEIXEIRA M.M., et al. Paracoccidioides lutzii sp. nov: biological and clinical implications. Med Mycol. v.52,n.1,p.19-28, 2014 
Lauana Aparecida Santos

Doutoranda do Programa de Pós-graduação em

Ciências Farmacêuticas - UNIFAL

Julianne Caravita Grisolia

Doutoranda do Programa de Pós-graduação em

Biociências - UNIFAL

Adriano Macedo de Oliveira

Professor e Médico Patologista do Hospital

Universitário Alzira Velano - UNIFENAS 